Need for sexual and reproductive education in high school teens

\title{
Necesidad de educación sexual y reproductiva en adolescentes de escuela secundaria
}

\author{
María B. Sánchez-Rojas, ${ }^{1}$ Sandra O. Gutiérrez-Enríquez,, ${ }^{2}$ Darío Gaytán-Hernández,, ${ }^{2}$ Yolanda Terán-Figueroa²
}

\begin{abstract}
Introduction: adolescents face problems that threaten their health, among which the adolescent arm and sexually transmitted infections, both preventable with education, stand out. Objective: identify the level of need for sexual and reproductive education in high school adolescents.

Material and methods: cross-sectional study, conducted from August 2018 to March 2019. Sample of 227 students from a public high school in the state of San Luis Potosí, enrolled in the 2018-2019 school year. A self-administered survey was applied to measure the need and an ordinal scale was designed based on the score obtained: high $=73-93$, medium $=52-72$ and low $=31-51$. The X2 test was applied to determine association between variables.

Results: most students have a medium level of need for sexual and reproductive education. Second grade students have a greater need for education $(35.2 \%)$ than first $(16.7 \%)$ and third $(6 \%)$ students with a significant difference $(\mathrm{p}<0.001)$. Those who have less need for sex education are those who talk frequently with their parents.

Conclusion: It is necessary that sexual education be comprehensive and taught from basic education levels on an ongoing basis, to train parents so they can provide better information. Likewise, encourage communication between parents and adolescent.
\end{abstract}

Keywords: sex education, sexual health, adolescent, adolescent health.

Citación: Sánchez Rojas MB., Gutiérrez Enríquez SO., Gaytán Hernández D., Terán Figueroa

Y. Necesidad de educación sexual y reproductiva en adolescentes de escuela secundaria, Rev Enferm Neurol. 2020;19(1):pp. 15-24.

Correspondencia: Sandra Olimpia Gutiérrez Enríquez.

E-mail: sgutierr01@gmail.com

${ }^{1}$ Licenciada en Enfermería

${ }^{2}$ Profesor (a) investigador (a) en la Facultad de

Enfermería y Nutrición, UASLP.
Recibido: 11 de octubre 2019

Aceptado: 20 de diciembre 2019 


\section{Resumen}

Introdución: los adolescentes enfrentan problemáticas que atentan su salud, entre los que destacan el embrazo y las infecciones de transmisión sexual, ambas prevenibles con educación.

Objetivo: identificar el nivel de necesidad de educación sexual y reproductiva en adolescentes de secundaria.

Material y métodos: estudio transversal, realizado de agosto 2018 a marzo 2019. Muestra de 227 estudiantes de una secundaria pública del estado de San Luis Potosí, inscritos en el ciclo escolar 2018-2019. Se aplicó una encuesta autoadministrada para medir la necesidad y se diseñó una escala ordinal con base al puntaje obtenido: $a l t a=73-93$, media $=52-72$ y baja $=31-51$. Se aplicó la prueba $\mathrm{X} 2$ para determinar asociación entre variables.

Resultados: la mayoría de los alumnos tienen un nivel de necesidad de educación sexual y reproductiva medio. Los estudiantes de segundo grado tienen mayor necesidad de educación (35.2\%) que los de primero $(16.7 \%)$ y tercero $(6 \%)$ con una diferencia significativa $(\mathrm{p}<0.001)$. Quienes tienen menor necesidad de educación sexual son aquellos que platican frecuentemente con sus padres.

Conclusión: es necesario que la educación sexual sea integral e impartida desde niveles de educación básica de manera continua; asimismo, capacitar a los padres de familia para que puedan brindar más información. De igual forma, fomentar la comunicación entre padres e hijos.

Palabras clave: educación sexual, salud sexual, adolescente, salud del adolescente.

\section{Introducción}

La adolescencia es una etapa donde los jóvenes se encuentran en riesgo de enfrentar problemas que pueden afectar su salud y desarrollo personal, entre estos se encuentra el embarazo y las infecciones de transmisión sexual (ITS). El Fondo de Población de las Naciones Unidas (FPNU) ha informado que todos los días 20,000 mujeres menores de 18 años dan a luz en países en vías de desarrollo; y de los 7,3 millones de partos de adolescentes que ocurren cada año, corresponden a niñas menores de 15 años. ${ }^{1}$ Además, las complicaciones durante el embarazo y parto son la segunda causa de muerte entre las jóvenes de 15 a 19 años, también, en este periodo de edad, cerca de tres millones de jóvenes recurren a abortos peligrosos que contribuyen al aumento de muertes maternas y a secuelas por enfermedades obstétricas. ${ }^{2}$ En cuanto a las ITS, en la población adolescente se estima que anualmente una de cada 20 jóvenes adolescentes contrae una infección bacteriana por contacto sexual y cada vez se presenta en edades más tempranas. ${ }^{3}$

México ocupa el primer lugar en embarazo adolescente según lo informa la Organización para la Cooperación y el Desarrollo Económicos (OCDE), ya que ocurren cerca 73.6 nacimientos por cada 1000 mujeres entre 15 y 19 años de edad, mientras que el promedio de todos los países que integran la OCDE es de 14.6 
nacimientos por cada 1000 mujeres. ${ }^{4}$ Las ITS, de igual manera, afectan el desarrollo de los adolescentes mexicanos, en 2012 se diagnosticaron 2,799 infecciones de VIH y 1,772 casos de SIDA en los jóvenes de 15 a 24 años de edad, con una razón de 4 contagios masculinos por cada mujer en el país. ${ }^{5}$ La Dirección General de Epidemiología de la Secretaria de Salud de México, en su reporte, informa que hay 21 casos de VIH en el estado de San Luis Potosí de los cuales 14 son varones y 7 mujeres, en comparación con el año pasado, en 2017 se tuvo el registro de 14 casos. $^{6}$

Una de las acciones necesarias para disminuir el número de casos de estos problemas, es a través de la educación sexual, definida por la Organización de las Naciones Unidas para la Educación, la Ciencia y la Cultura (UNESCO) como "un enfoque culturalmente relevante y apropiado a la edad del participante, que enseña sobre el sexo y las relaciones interpersonales a través del uso de información científicamente rigurosa, realista y sin prejuicios de valor". Asimismo, la UNESCO junto a otros organismos internacionales promulgó las Orientaciones Técnicas Internacionales sobre Educación en Sexualidad ${ }^{7}$ en 2017, donde indica 8 conceptos clave que integran la educación sexual, tales como: 1. relaciones, 2. valores, derechos, cultura y sexualidad, 3. cómo entender el género, 4 . la violencia y cómo mantenerse seguros, $\mathbf{5}$. habilidades para la salud y el bienestar, 6. el cuerpo humano y el desarrollo, 7. sexualidad y conducta sexual y $\mathbf{8}$. salud sexual y reproductiva, estos a su vez están constituidos por subtemas, los cuales conforme al rango de edad de las personas a las que vaya dirigido el programa educativo, desde los 5 años, hasta los 18 años o más; en conformidad con las orientaciones técnicas de educación sexual integral en el octavo concepto. Artículos recientes, revelan que los adolescentes de 12 a 15 años, deben de conocer la diversidad de preferencias sexuales, la diferencia entre género, sexo, actividad sexual, y reproducción, saber los cambios durante la adolescencia y pubertad, así como la importancia de la autoestima y los servicios brindados de forma gratuita para tratar aspectos psicológicos, de enterarse que algunas actitudes o sentimientos relacionados con la sexualidad son naturales, estar consciente de la importancia de respetar a los demás y a ellos mismos, tener conocimiento de la responsabilidad de su propia sexualidad, la importancia de la prevención e identificación de comportamientos sexuales que pongan en riesgo su salud y bienestar, las distintas formas de anticoncepción, al igual que, las ventajas y desventajas de cada uno y donde pueden recibir atención especializada. ${ }^{7-9}$

La educación sobre salud sexual y reproductiva es importante pues de esta manera los adolescentes tienen herramientas para decidir sobre su futuro, prevenir riesgos a su salud y vivir una vida plena. ${ }^{7}$ El objetivo de este estudio fue conocer la necesidad de educación sexual y reproductiva en adolescentes de una secundaria pública en el estado de San Luis Potosí, México.

\section{Material y métodos}

Estudio observacional, transversal, relacional y comparativo, realizado de agosto 2018 a marzo 2019. Universo de 451 estudiantes de una secundaria pública del estado de San Luis Potosí, inscritos en el ciclo escolar 2018-2019. Se utilizó la fórmula para cálculos de muestra con población finita, con un margen de error del $5 \%$ y un nivel de confianza del $95 \%$. El tamaño de muestra quedó integrado por 227 alumnos. Se aplicó una encuesta autoadministrada que fue elaborada ex profeso para medir el nivel de necesidad de educación sexual y 
reproductiva. Para fines de esta investigación, se definió este concepto como la insuficiencia del abordaje de temas de educación sexual y reproductiva (embarazo y prevención del embarazo, infecciones de transmisión sexual y conductas sexuales de riesgo), con base en el concepto 8 de salud sexual y reproductiva de las Orientaciones Técnicas Internacionales sobre Educación en Sexualidad de la UNESCO, y en la NORMA Oficial Mexicana NOM-047-SSA2-2015 para la atención a la salud del grupo etario de 10 a 19 años de edad. ${ }^{10}$ La encuesta está compuesta por tres apartados: I. Datos sociodemográficos, II. Embarazo y prevención del embarazo, infecciones de transmisión sexual y conductas sexuales de riesgo. III. Educación sexual y reproductiva de padres a hijos. Para medir la necesidad de educación se diseñó una escala ordinal para clasificar la necesidad con base al puntaje total (alta $=73-93$, media $=52-72$, Baja $=$ 31-51) del apartado II, con un total de 31 items Likert. Los participantes tacharon las opciones de acuerdo a la frecuencia de información que les han otorgado sobre alguno de los temas anteriores, de manera que la opción de "muchas veces" tuvo el valor de 1 punto (lo que significa menor necesidad de educación) y la opción "nunca" tuvo el valor máximo de 3 puntos (lo que representa una mayor necesidad de educación). La encuesta fue validada por expertos y por prueba piloto. Se aplicó una evaluación para valorar su confiabilidad (Alpha de Cronbach) la cual arrojó un valor de 0.91 . Se aplicó la prueba $\mathrm{X}^{2}$ para determinar diferencia significativa entre la variable de necesidad con datos sociodemográficos y temas de salud sexual y reproductiva, así como entre la frecuencia de educación sexual y reproductiva proporcionada de padres a hijos con el nivel de necesidad. Todos los análisis se realizaron en el programa estadístico Statical Package for the
Social Sciences (SPSS) versión 22. Este estudio estuvo apegado a la Ley General de Salud en Materia de Investigación, y el artículo $100^{\circ}$, fracción IV de la declaración de Helsinki, ${ }^{11}$ por tanto, todos los participantes tuvieron el consentimiento de sus padres para participar en el estudio. El proyecto obtuvo registro de un Comité de Ética Universitario (CEIFE-2019-279). Los autores declaran no tener conflicto de intereses.

\section{Resultados}

Se obtuvo que tanto mujeres (61.6\%) como hombres $(56.9 \%)$ tienen necesidad media de educación sexual y reproductiva. El rango de edad con la necesidad más alta fue de 13 a 14 años $(20.9 \%)$. Se encontró una diferencia significativa $\left(p^{*}=<0.001\right)$ entre los estudiantes de cada grado escolar y el nivel de necesidad de educación sexual y reproductiva, donde los alumnos de segundo grado tienen mayor necesidad de educación sexual y reproductiva $(35.2 \%)$ a diferencia de los alumnos de primer grado (16.7\%) y de tercero (6\%). La mayoría de los estudiantes que tenían pareja tienen mayor necesidad de educación sexual y reproductiva (19.3) que aquellos que no tienen pareja (18). La mayoría de los estudiantes tienen como única ocupación el estudio (tabla 1).

Ambos sexos presentan necesidad alta de educación sobre métodos anticonceptivos (hombres 39.5\%, mujeres 39.7\%). Las mujeres tienen necesidad baja de educación sobre embarazo adolescente con el $65.6 \%$ y en un menor porcentaje también los hombres con el $48 \%$ (tabla 2).

Se encontró una diferencia significativa $(p=<0.001)$ entre el nivel de necesidad de educación sexual reproductiva y la frecuencia en que los padres hablan con sus hijos sobre sexualidad. Se observó que los padres hablan con menor 
Tabla 1. Nivel de necesidad de educación sexual, reproductiva y características sociodemográficas. Estudiantes de secundaria. San Luis Potosí, S.L.P., 2019.

$\mathrm{n}=227$

\begin{tabular}{|c|c|c|c|c|c|c|c|c|c|c|}
\hline \multirow{3}{*}{ Datos sociodemográficos } & \multicolumn{10}{|c|}{ Nivel de la necesidad de educación sexual y reproductiva } \\
\hline & \multicolumn{2}{|c|}{ Baja } & \multicolumn{2}{|c|}{ Media } & \multicolumn{2}{|r|}{ Alta } & \multirow{2}{*}{ Total } & \multirow{2}{*}{ g.l. ${ }^{* *}$} & \multirow{2}{*}{$\mathrm{X}^{2}$} & \multirow{2}{*}{ Valor de $\mathrm{p}$} \\
\hline & $F_{x}$ & $\%$ & $F_{x}$ & $\%$ & $\mathrm{Fx}_{\mathrm{x}}$ & $\%$ & & & & \\
\hline $\begin{array}{l}\text { Sexo del encuestado } \\
\text { Mujer } \\
\text { Hombre }\end{array}$ & $\begin{array}{l}28 \\
22\end{array}$ & $\begin{array}{l}22.4 \\
21.6\end{array}$ & $\begin{array}{l}77 \\
58\end{array}$ & $\begin{array}{l}61.6 \\
26.9\end{array}$ & $\begin{array}{l}20 \\
22\end{array}$ & $\begin{array}{c}16 \\
21.6\end{array}$ & $\begin{array}{l}125 \\
102\end{array}$ & 2 & 1.1712 & 0.557 \\
\hline $\begin{array}{l}11-12 \\
13-14 \\
15-16\end{array}$ & $\begin{array}{c}14 \\
27 \\
9\end{array}$ & $\begin{array}{c}24.6 \\
19.4 \\
29\end{array}$ & $\begin{array}{l}32 \\
83 \\
20\end{array}$ & $\begin{array}{l}56.1 \\
59.7 \\
64.5 \\
\end{array}$ & $\begin{array}{c}11 \\
29 \\
2\end{array}$ & $\begin{array}{c}19.3 \\
20.9 \\
6.5\end{array}$ & $\begin{array}{c}57 \\
139 \\
31\end{array}$ & 4 & 4.396 & 0.355 \\
\hline $\begin{array}{l}\text { Primero } \\
\text { Segundo } \\
\text { Tercero }\end{array}$ & $\begin{array}{l}16 \\
12 \\
22\end{array}$ & $\begin{array}{l}22.2 \\
16.9 \\
26.2\end{array}$ & $\begin{array}{l}44 \\
34 \\
57\end{array}$ & $\begin{array}{l}61.1 \\
47.9 \\
67.9\end{array}$ & $\begin{array}{c}12 \\
25 \\
5\end{array}$ & $\begin{array}{c}16.7 \\
35.2 \\
6\end{array}$ & $\begin{array}{l}72 \\
71 \\
84\end{array}$ & 4 & 22.132 & $0.000^{*}$ \\
\hline $\begin{array}{r}\text { Situación sentimental } \\
\text { Con novia/o } \\
\text { Sin novia/o }\end{array}$ & $\begin{array}{l}19 \\
31\end{array}$ & $\begin{array}{l}21.6 \\
22.3\end{array}$ & $\begin{array}{l}52 \\
83\end{array}$ & $\begin{array}{l}59.1 \\
59.7\end{array}$ & $\begin{array}{l}17 \\
25\end{array}$ & $\begin{array}{c}19.3 \\
18\end{array}$ & $\begin{array}{c}88 \\
139\end{array}$ & 2 & 0.068 & 0.967 \\
\hline $\begin{array}{l}\text { Estudia } \\
\text { Estudia y } \\
\text { trabaja }\end{array}$ & $\begin{array}{l}16 \\
12 \\
22\end{array}$ & $\begin{array}{l}22.2 \\
16.9 \\
26.2\end{array}$ & $\begin{array}{l}44 \\
34 \\
57\end{array}$ & $\begin{array}{l}61.1 \\
47.9 \\
67.9\end{array}$ & $\begin{array}{c}12 \\
25 \\
5\end{array}$ & $\begin{array}{c}16.7 \\
35.2 \\
6\end{array}$ & $\begin{array}{l}72 \\
71 \\
84\end{array}$ & 4 & 22.132 & $0.000 *$ \\
\hline
\end{tabular}

$* *$ g.l. $=$ grados de libertad $*_{\mathrm{p}}<0.001$

Fuente: Encuesta de necesidades de educación sexual y reproductiva 2019

Tabla 2. Necesidades de educación sexual y reproductiva de acuerdo al sexo. Estudiantes de secundaria. San Luis Potosí, S.L.P., 2019.

$\mathrm{n}=227$

\begin{tabular}{|c|c|c|c|c|c|c|c|c|c|}
\hline \multirow{3}{*}{ Sexo } & \multicolumn{9}{|c|}{ Nivel de la necesidad de educación sexual y reproductiva } \\
\hline & \multicolumn{2}{|c|}{ Baja } & \multicolumn{2}{|c|}{ Media } & \multicolumn{2}{|c|}{ Alta } & \multirow{2}{*}{ g.l.** } & \multirow{2}{*}{$\mathrm{X}^{2}$} & \multirow{2}{*}{ Valor de $\mathrm{p}$} \\
\hline & $\mathrm{Fx}_{\mathrm{x}}$ & $\%$ & $\mathrm{Fx}_{\mathrm{x}}$ & $\%$ & $F_{x}$ & $\%$ & & & \\
\hline \multicolumn{7}{|c|}{ Necesidad de educación sobre el embarazo } & \multirow{3}{*}{2} & \multirow[b]{3}{*}{9.870} & \multirow{3}{*}{0.007} \\
\hline Mujer & 28 & 22.4 & 77 & 61.6 & 20 & 16 & & & \\
\hline Hombre & 22 & 21.6 & 58 & 26.9 & 22 & 21.6 & & & \\
\hline \multicolumn{7}{|c|}{ Necesidad de educación sobre Métodos anticonceptivos } & \multirow{3}{*}{2} & \multirow{3}{*}{0.582} & \multirow{3}{*}{0.748} \\
\hline Mujer & 20 & 16.5 & 53 & 43.8 & 48 & 39.7 & & & \\
\hline Hombre & 13 & 13.1 & 100 & 45.5 & 87 & 39.5 & & & \\
\hline \multicolumn{7}{|c|}{ Necesidad de educación sobre Infecciones de Trasmisión Sexual } & \multirow{3}{*}{2} & \multirow{3}{*}{0.311} & \multirow{3}{*}{0.856} \\
\hline Mujer & 36 & 28.8 & 67 & 53.6 & 22 & 17.6 & & & \\
\hline Hombre & 31 & 30.4 & 51 & 50 & 20 & 19.6 & & & \\
\hline \multicolumn{7}{|c|}{ Necesidad de educación sobre Conductas Sexuales de Riesgo } & \multirow{3}{*}{2} & \multirow{3}{*}{2.213} & \multirow{3}{*}{0.331} \\
\hline Mujer & 44 & 35.2 & 61 & 48.8 & 20 & 16 & & & \\
\hline Hombre & 27 & 26.5 & 54 & 52.9 & 21 & 20.6 & & & \\
\hline
\end{tabular}

Fuente: Encuesta de necesidades de educación sexual y reproductiva 2019 
frecuencia acerca de la prevención de infecciones de transmisión sexual con sus hijos $(59.4 \%)$ y de la prevención de conductas sexuales de riesgo (57.1\%) y hablan más sobre la prevención del embarazo adolescente $(80 \%)$ y el uso de métodos anticonceptivos (74\%) (tabla 3). Se reveló una diferencia significativa $(\mathrm{p}=<0.001)$ entre el sexo de los participantes y la frecuencia con la que recibían educación sexual y reproductiva, por parte de sus padres. Los participantes de 11 años de edad recibían con constancia información sobre educación sexual y reproductiva de parte de sus padres (100\%), a diferencia de los participantes de 16 años que era poco frecuente que recibieran información de sus padres de educación sexual y reproductiva. Para el $47.2 \%$ de los alumnos de primer grado es poco frecuente que sus padres hablen con ellos sobre sexualidad, al igual que para el 38\% de los alumnos de segundo y el $42.9 \%$ de tercero (42.9\%) (tabla 4 ).

Tabla 3. Nivel de necesidad de educación de los participantes y frecuencia de educación sexual y reproductiva proporcionada por padres a hijos. Estudiantes de secundaria. San Luis Potosí. S.L.P., 2019.

$\mathrm{n}=227$

\begin{tabular}{|c|c|c|c|c|c|c|c|c|c|}
\hline \multirow{3}{*}{$\begin{array}{l}\text { Nivel de necesidad de } \\
\text { educación sexual } \\
\text { y reproductiva }\end{array}$} & \multicolumn{9}{|c|}{$\begin{array}{l}\text { Frecuencia de educación sexual y reproductiva proporcionada por } \\
\text { padres a hijos }\end{array}$} \\
\hline & \multicolumn{2}{|c|}{ Muchas veces } & \multicolumn{2}{|c|}{ A veces } & \multicolumn{2}{|c|}{ Nunca } & \multirow{2}{*}{ g.l. $* *$} & \multirow{2}{*}{$\mathrm{X}^{2}$} & \multirow{2}{*}{ Valor de 1} \\
\hline & $\mathrm{Fx}$ & $\%$ & $\mathrm{Fx}_{\mathrm{x}}$ & $\%$ & $\mathrm{Fx}$ & $\%$ & & & \\
\hline \multicolumn{10}{|l|}{ Prevención del embarazo } \\
\hline Alta & 6 & 14.3 & 22 & 52.4 & 14 & 33.3 & & & \\
\hline media & 54 & 40 & 60 & 44.4 & 21 & 15.6 & 4 & 44.991 & $0.000^{*}$ \\
\hline Baja & 40 & 80 & 70 & 14 & 3 & 6 & & & \\
\hline \multicolumn{7}{|c|}{ Uso de métodos anticonceptivos } & & & \\
\hline Alta & 3 & 7.1 & 16 & 40.5 & 22 & 52.4 & & & \\
\hline media & 34 & 25.2 & 64 & 47.4 & 37 & 27.4 & 4 & 61.733 & $0.000^{*}$ \\
\hline Baja & 37 & 66 & 10 & 20 & 3 & 6 & & & \\
\hline \multicolumn{7}{|c|}{ Prevención de infecciones de transmisión sexual } & & & \\
\hline Alta & 2 & 4.8 & 15 & 35.7 & 25 & 59.5 & & & \\
\hline media & 40 & 29.6 & 54 & 40 & 41 & 30.4 & 4 & 48.737 & $0.000 *$ \\
\hline Baja & 33 & 66 & 13 & 26 & 4 & 8 & & & \\
\hline \multicolumn{7}{|c|}{ Prevención de conductas sexuales de riesgo } & & & \\
\hline Alta & 3 & 7.1 & 15 & 35.7 & 24 & 57.1 & & & \\
\hline media & 38 & 28.1 & 44 & 32.6 & 53 & 39.3 & 4 & 32.218 & $0.000 *$ \\
\hline Baja & 27 & 54 & 18 & 36 & 5 & 10 & & & \\
\hline
\end{tabular}

Fuente: Encuesta de necesidades de educación sexual y reproductiva 2019

\section{Discusión}

De acuerdo a los Objetivos de Desarrollo Sostenible ${ }^{12}$ y la Estrategia Mundial para la Salud de la Mujer, el Niño y el Adolescente
$(2016-2030)^{13}$ es imprescindible brindar educación sanitaria, información y servicios de salud sexual y reproductiva, para lograr disminuir los embarazos adolescentes y las infecciones de transmisión sexual, al igual que los problemas derivados de ellos como: muertes maternas, alto 
índice de personas infectadas con VIH o SIDA, marginación, pobreza, deserción escolar, entre otras. La UNESCO, en conjunto con otras organizaciones internacionales, creó las Orientaciones Técnicas Internacionales sobre Educación en Sexualidad dirigido a escuelas, profesores y educadores de salud y menciona que la educación sexual y reproductiva tiene efectos benéficos para los adolescentes, pues representa un mayor conocimiento para los jóvenes, contribuye al mejoramiento de sus actitudes y conductas en relación con la salud sexual y reproductiva.?

En este estudio la falta de educación sexual y reproductiva de la mayoría de los estudiantes es media, esto da a entender que, a pesar de recibir algún tipo de información básica, siguen teniendo deficiencias que los ponen en riesgo de presentar algún embarazo adolescente, o de contraer alguna ITS. La mayoría de los estudiantes que indicaron tener una relación sentimental, tienen mayor carencia de educación sexual, esto los coloca en riesgo de iniciar vida sexual activa sin las herramientas indispensables para tener una vida sexual responsable y saludable. En investigaciones recientes como la de Sandres en Brasil, 2017, se reportaron casos de adolescentes que inician relaciones sexuales entre los 12 y 15 años, un dato importante, de acuerdo a este estudio, no cuentan aún con la preparación e información suficiente para ejercer una sexualidad responsable. ${ }^{14}$ En el estudio realizado por Peralta en México, 2018, el 25\% de los adolescentes entre 15 y 17 años, ya habían iniciado vida sexual, además, se señala que casi la mitad de los adolescentes siguen teniendo información errónea acerca de la sexualidad, por ejemplo: las prácticas no funcionales para evitar un embarazo, las duchas vaginales poscoito. ${ }^{15}$ En esta

Tabla 4. Frecuencia de educación sexual y reproductiva proporcionada por padres a hijos y datos sociodemográficos. Estudiantes de secundaria. San Luis Potosí, S.L.P., 2019.

$\mathrm{n}=227$

\begin{tabular}{|c|c|c|c|c|c|c|c|c|c|}
\hline \multirow{3}{*}{$\begin{array}{c}\text { Datos } \\
\text { sociodemográficos }\end{array}$} & \multicolumn{9}{|c|}{$\begin{array}{l}\text { Frecuencia de educación sexual y reproductiva proporcionada por } \\
\text { padres a hijos }\end{array}$} \\
\hline & \multicolumn{2}{|c|}{ Muchas veces } & \multicolumn{2}{|c|}{ A veces } & \multicolumn{2}{|c|}{ Nunca } & \multirow{2}{*}{ g.l.** } & \multirow{2}{*}{$\mathrm{X}^{2}$} & \multirow{2}{*}{ Valor de $\mathrm{p}$} \\
\hline & $\mathrm{Fx}$ & $\%$ & $\mathrm{Fx}$ & $\%$ & $F_{x}$ & $\%$ & & & \\
\hline Sexo & & & & & & & \multirow{3}{*}{2} & \multirow{3}{*}{13.502} & \multirow{3}{*}{0.001} \\
\hline Mujer & 634 & 50.4 & 26 & 20.8 & 36 & 28.8 & & & \\
\hline Hombre & 34 & 33.3 & 44 & 43.1 & 24 & 23.5 & & & \\
\hline Edad & & & & & & & \multirow{7}{*}{10} & \multirow{7}{*}{10.472} & \multirow{7}{*}{0.400} \\
\hline 11 & 0 & 0 & 0 & 0 & 2 & 100 & & & \\
\hline 12 & 25 & 45.5 & 17 & 30.9 & 13 & 23.6 & & & \\
\hline 13 & 24 & 42.1 & 16 & 28.1 & 17 & 29.8 & & & \\
\hline 14 & 35 & 42.7 & 25 & 30.5 & 22 & 26.8 & & & \\
\hline 15 & 11 & 37.9 & 12 & 41.4 & 6 & 20.7 & & & \\
\hline 16 & 2 & 100 & 0 & 0 & 0 & 0 & & & \\
\hline Grado escolar & & & & & & & \multirow{4}{*}{4} & \multirow{4}{*}{2.208} & \multirow{4}{*}{0.698} \\
\hline Primer grado & 34 & 47.2 & 23 & 31.9 & 15 & 20.8 & & & \\
\hline Segundo grado & 27 & 38 & 22 & 31 & 22 & 31 & & & \\
\hline Tercer grado & 36 & 42.9 & 25 & 29.8 & 23 & 27.4 & & & \\
\hline
\end{tabular}

$* *$ g.l. = grados de libertad $*_{\mathrm{p}}<0.001$

Fuente: Encuesta de necesidades de educación sexual y reproductiva 2019 
investigación algunos participantes no habían escuchado hablar de algún tipo de conductas sexuales de riesgo, algunas infecciones de trasmisión sexual y algunos métodos anticonceptivos. Asimismo, expresaron la importancia de hablar de estos temas, por parte de los adultos, sin vergüenza y ser escuchados para aclarar sus dudas.

Los estudiantes que tenían mayor necesidad de educación sexual fueron aquellos que tenían 13 y 14 años, estudiantes, donde la mayoría cursa el segundo grado de secundaria, es fundamental aclarar, en este grado escolar y en primer año es donde se abordan temas de prevención de embarazo, uso de métodos anticonceptivos, prevención de conductas sexuales de riesgo e infecciones de transmisión sexual en las materias de biología, cívica y ética en la escuela pública, respecto a esto es crucial mencionar que desde 1974 se han incluido temas sobre sexualidad en los libros de texto gratuito que otorga el gobierno de México, con enfoque al uso de métodos anticonceptivos y a la prevención de embarazos; sin embargo, la información que contienen estos libros no es educación sexual integral, a partir de la nueva reforma educativa del gobierno actual, incluye temas de diversidad sexual. ${ }^{16}$ En un estudio realizado por Obach, et al., en Chile, 2017, los estudiantes declararon que la información que recibían de la escuela era muy básica pues se centraba en hablar de aspectos biológicos y genitales. Los autores refieren que es necesario que se trabaje de la mano entre el sector salud y educativo en suma coordinación ${ }^{17}$

En el estudio de Orcasita, et al., en 2018 se encontró que los padres usualmente transmiten información de acuerdo a sus experiencias, además se enfocan en discursos sobre la prevención de embarazos, excluyendo los temas de prevención de infecciones de transmisión sexual, conductas sexuales de riesgo y diversidad sexual ${ }^{18}$. En contraste, en el estudio de M. Grossman en Estados Unidos de Norteamérica, menciona que puede influir en el comportamiento sexual de los adolescentes la información proporcionada por los padres ${ }^{19}$, por lo que, incluir a estos dentro de los programas de salud sexual y reproductiva sería una pieza clave.

Resulta relevante que exista mayor necesidad de educación sexual y reproductiva en adolescentes hombres, sobre embarazo adolescente que en mujeres, pues es a ellas a quienes más se dirige esta información, de acuerdo al estudio de Orcasita, en Colombia, los hombres reciben menos información sobre sexualidad, a diferencia de las mujeres, señalando la carencia de programas educativos con perspectiva de género. $^{20}$

\section{Conclusiones}

La mayoría de los participantes siguen teniendo necesidades de educación sexual y reproductiva, los alumnos con mayor necesidad de educación son los de segundo grado de 13 a 14 años, los padres platican más con sus hijos sobre prevención del embarazo y el uso de métodos anticonceptivos y menos de la prevención de ITS y conductas sexuales de riesgo. Es fundamental reforzar la educación sexual, a través del diseño de talleres para padres, demostraciones y situaciones vivenciales con pares dirigido a los alumnos, con la finalidad de que puedan brindar a los adolescentes una orientación completa y eficaz. El personal de salud debe explorar otras formas de orientar, menos tradicionales y más innovadoras que despierten la consciencia de los adolescentes. Para futuras investigaciones se recomienda realizar estudios cualitativos para conocer las necesidades de educación percibidas 
por los alumnos, padres, maestros y profesionales de salud, así como estudios enfocados en conocimientos de padres sobre estos temas. Incluso es imprescindible la evaluación de los programas del sector salud y educativo, con base en las Orientaciones Técnicas de Educación Sexual Integral de la UNESCO.

\section{Referencias}

1. Fondo de Población de las Naciones Unidas. Maternidad en la niñez: enfrentar el reto del embarazo en adolescentes. Estado de la población mundial. UNFPA; 2013. 132. Disponible en: http://bit.ly/38w2u09 [Acceso: 25 /09/2018]

2. OMS. El embarazo en la adolescencia. Organización Mundial de la Salud. Febrero 2018. Disponible en: http://bit.ly/2wzYzlK [Acceso: 19/09/18]

3. OMS. Diez datos sobre las enfermedades de transmisión sexual. Organización Mundial de la Salud. Febrero 2018. Disponible en: http://bit. ly/2THVMy'T [Acceso: 20/09/2018]

4. Mabel Gabriel, Patrick Lenain. Mejorando las oportunidades de las mujeres en México. OCDE. Disponible en: http://bit.ly/2TFE5QG [Acceso: 21/09/18]

5. CENSIDA. (2014). Programa Especial del VIH, SIDA e ITS, 2013-2018 México: Centro Nacional para la Prevención y el Control del VIH y el SIDA. Subsecretaría de Prevención y Promoción de la Salud. Disponible en: http://bit.ly/39vfeFY [Acceso: 25/09/2018]

6. S.L.P. registra aumento en enfermedades venéreas. Pulso Diario de San Luis. 2018. Disponible en: http://bit.ly/2IqiCFX [Acceso: 01/10/2018]

7. UNESCO. Orientaciones técnicas internacionales sobre educación en sexualidad. Organización de las Naciones Unidas para la Educación la Ciencia y la Cultura. 2018. Disponible en: http://bit.ly/2x6tPJp [Acceso: 01/11/2018]

8. Cryan G, Cimas M. Acceso a información vinculada a sexualidad a través del ámbito educativo y las TIC. Ciencia, Docencia y
Tecnología. 2018, 29(57), 256-271. Disponible en: http://bit.ly/3auBBuZ [Acceso: 26/08/2019]

9. Callejón Chinchilla MD, Gila-Ordóñez JM. Necesidad de trabajar las relaciones de pareja saludable desde contextos socio-educativos. Sophia 2018;14(1):31-8. Disponible en: http:// bit.ly/2vLveVB [Acceso: 26/08/2019].

10. Norma Oficial Mexicana NOM-047SSA2-2015 [en línea]. Para la atención a la salud del grupo etario de 10 a 19 años de edad. Diario Oficial de la Federación. 20 feb 2015. Disponible en: http://bit.ly/3cyepxS [Acceso: 16/10/2018]

11. Declaración de Helsinki de la Asociación Médica Mundial. Comisión Nacional de Arbitraje Médico. Disponible en: http:/ / bit.ly/2PRQEqW [Acceso: 23/09/2018].

12. PNUD. Objetivos del desarrollo sostenible (s.f.) Programa de las Naciones Unidas para el Desarrollo. Disponible en: http://bit.ly/2wuei67 [Acceso: 01/04/2019]

13. Todas las mujeres, todos los niños. Estrategia mundial para la salud de la mujer, el niño y el adolescente (2016-2030). 2015. Organización de las Naciones Unidas. Disponible en: http://bit. ly/3aqsSKm [Acceso: 29/09/2018]

14. Sandres Lins L, Airton L, Gomes dos Santos R, Duarte de Morais T, de Andrade Beltrão T, de Lima Castro F. Análise do comportamento sexual de adolescentes. Rev Brasileira em Promoção da Saúde 2017;30:47-56. Disponible en: http:/ /bit.ly/3axxu1w [Acceso: 22/02/2019]

15. Peralta-Sánchez A. Conocimientos y actitudes de 700 adolescentes de 12-17 años acerca de la sexualidad y la anticoncepción, originarios del sureste del estado de Puebla, México. Ginecol Obstet Mex 2018;86(9):606-10. Disponible en: https://doi.org/10.24245/gom.v86i9.2296 [Acceso: 22/02/2019]

16. Garduño V. Educación sexual: una polémica persistente. Red del Instituto Nacional para la Evaluación de la Educación, 2018. Disponible en: http://bit.ly/39tPvxo [Acceso: 28/03/2019]

17. Obach A, Sadler M, Jofré N. Salud sexual y reproductiva de adolescentes en Chile: el rol de la educación sexual. Presentado como ponencia en el Simposio: "Desigualdad, Género y Salud", en el marco 
del V Congreso de la Asociación Latinoamericana de Antropología y el XVI Congreso de Antropología en Colombia, celebrados en la Universidad Pontificia Javeriana, en la ciudad de Bogotá, del 6 al 9 de junio de 2017. Rev Sal Púb 2017;19(6):848-54. Disponible en: https://doi. org/10.15446/rsap.V19n6.70023 [01/03/2019]

18. Orcasita LT, Cuenca J, Montenegro JL, Garrido D, Haderlein A. Diálogos y saberes sobre sexualidad de padres con hijos e hijas adolescentes escolarizados. Rev Colomb Psicol 2018;27:41-53. Disponible en: https://doi.org/10.15446/rcp. v27n1.62148 [Acceso: 15/03/2019]
19. Grossman J, Lynch A, Richer A, DeSouza L, Ceder I. Extended-family talk about sex and teen sexual behavior. Inter J Environ Res Pub Health 2019;(3):480. Available from: https:// doi.org/10.3390/ijerph16030480 [Access: 2019 Apr 1]

20. Orcasita LT, Palma D, Sadeghian HC, Villafañe A, Sánchez DM, Sevilla TM, et al. Sexualidad en hombres adolescentes escolarizados: un análisis comparativo entre instituciones públicas y privadas en Colombia. Rev Cien Sal 2018;16(3):408-28. Disponible en: http://bit.ly/2vyBrEn [Acceso: 01/04/2019] 